Supporting Information

\title{
Band Engineering and Thermoelectric Performance Optimization of p-Type GeTe-Based Alloys through Ti/Sb \\ Co-doping
}

Luo Yue ${ }^{\mathrm{a}}$, Wenlin Cui ${ }^{\mathrm{a}}$, Shuqi Zheng ${ }^{\mathrm{a}, *}$, Yue Wu ${ }^{\mathrm{a}}$, Lijun Wang ${ }^{\mathrm{a}}$, Pengpeng Bai ${ }^{\mathrm{b}}$, Ximeng

Dong $^{\mathrm{a}}$

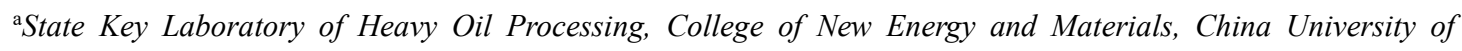
Petroleum, Beijing 102249, PR China

${ }^{\mathrm{b}}$ Department of Mechanical Engineering, State Key Laboratory of Tribology, Tsinghua University, Beijing, 100084, PR China

*Corresponding author. Tel.: +86 01089733200 ; Fax: +86 01089733973

E-mail address: zhengsq09@163.com 

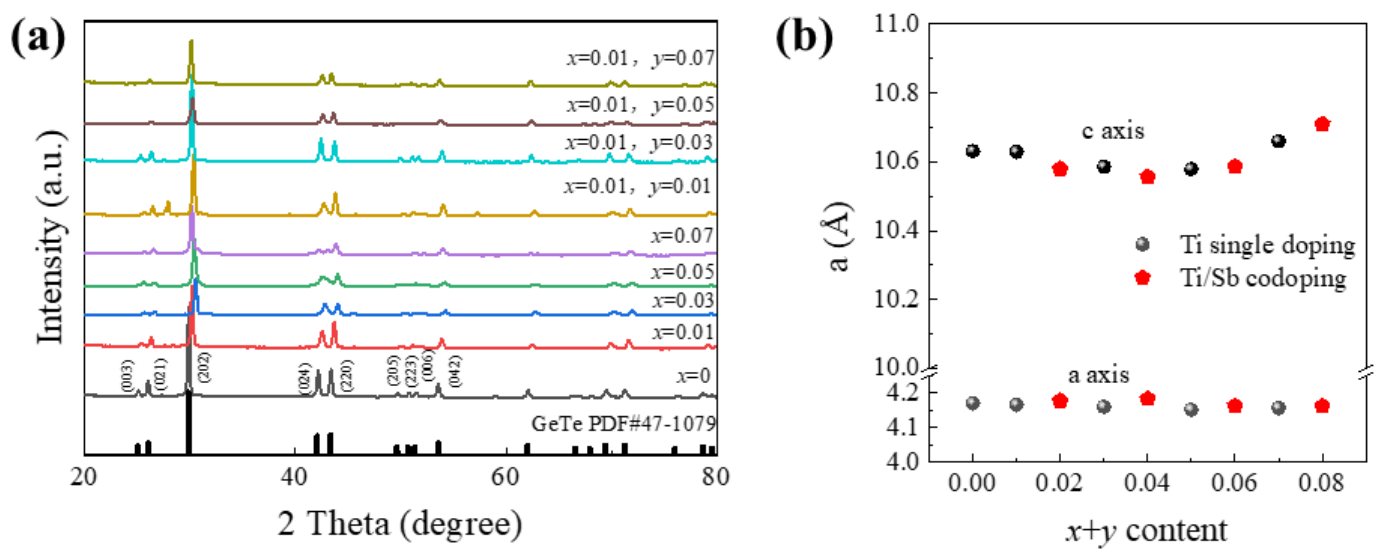

Figure $\mathrm{S} 1$ (a) XRD patterns of $\mathrm{Ge}_{1-x-y} \mathrm{Ti}_{x} \mathrm{Sb}_{y} \mathrm{Te}(x=0,0.01,0.03,0.05,0.07, y=0$, $0.01,0.03,0.05,0.07)$ samples at room temperature; (b) Ti and Sb content dependence of lattice parameters. 


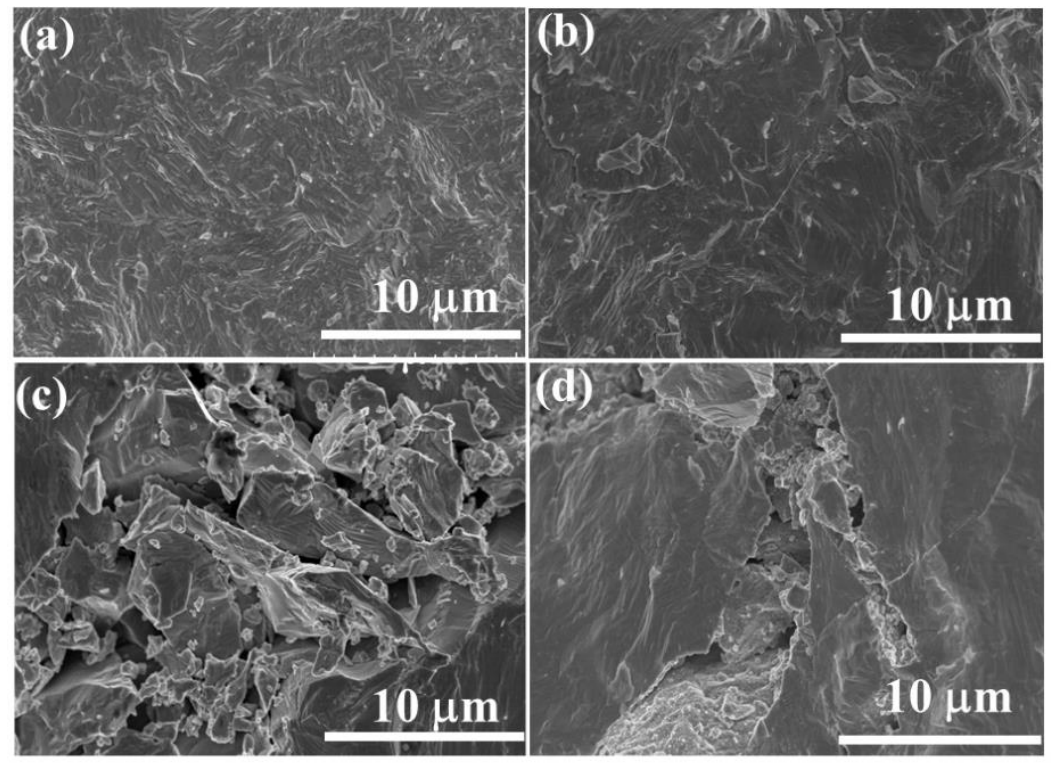

Figure S2 the fractured surface SEM images of $\mathrm{Ge}_{1-x} \mathrm{Ti}_{x} \mathrm{Te}(x=0.01,0.03,0.05$ and 0.07) (a) $\mathrm{Ge}_{0.99} \mathrm{Ti}_{0.01} \mathrm{Te}$, (b) $\mathrm{Ge}_{0.97} \mathrm{Ti}_{0.03} \mathrm{Te}$, (c) $\mathrm{Ge}_{0.95} \mathrm{Ti}_{0.05} \mathrm{Te}$ and (d) $\mathrm{Ge}_{0.95} \mathrm{Ti}_{0.05} \mathrm{Te}$. 


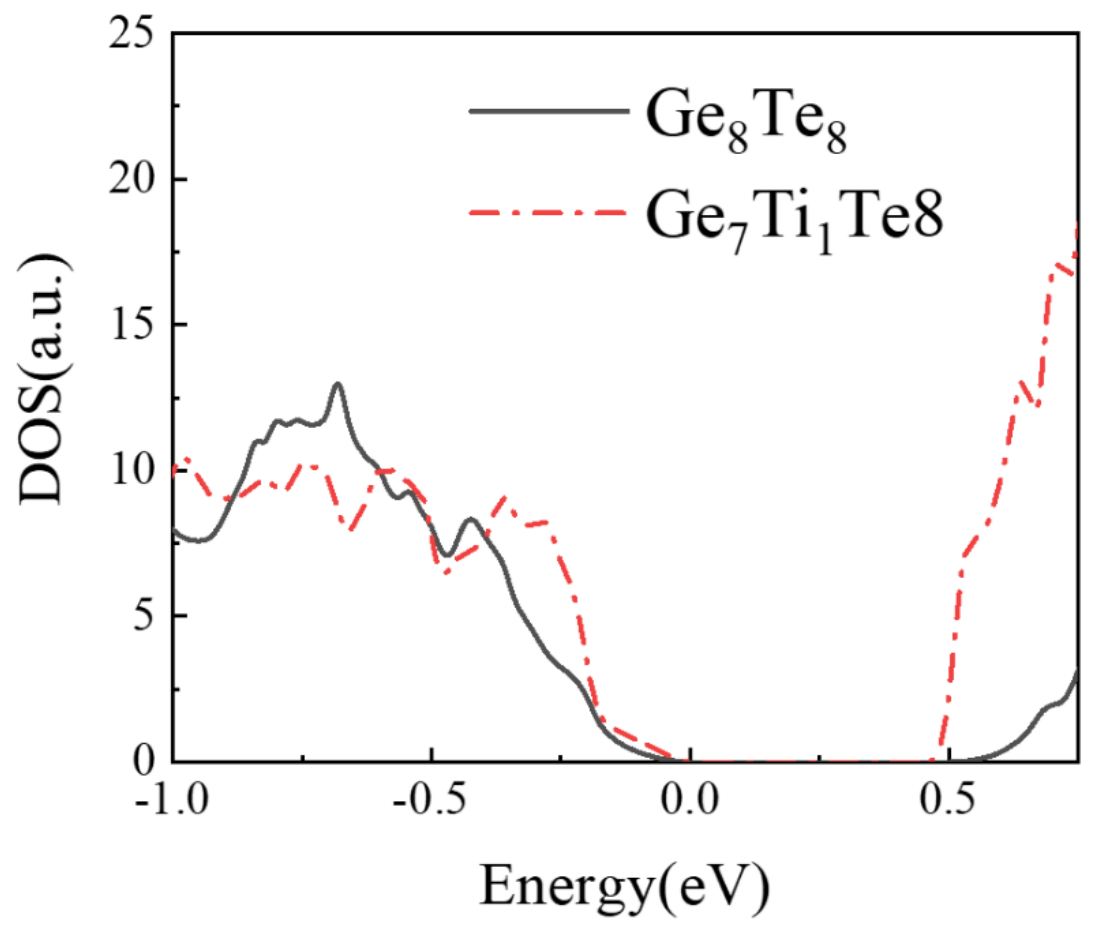

Figure $\mathrm{S} 3$ The DOS of $\mathrm{Ge}_{8} \mathrm{Te}_{8}$ and $\mathrm{Ge}_{7} \mathrm{Te}_{1} \mathrm{Te}_{8}$ 
Table R1. Room temperature transport parameter of $\mathrm{Ge}_{1-x} \mathrm{Ti}_{x} \mathrm{Te}(x=0.01,0.03,0.05$ and 0.07 )

\begin{tabular}{|c|c|c|c|c|c|}
\hline Sample & Composition & $\begin{array}{l}\text { Seebeck } \\
\left(\mu \mathrm{VK}^{-1}\right)\end{array}$ & $\begin{array}{c}\text { Electrical } \\
\text { conductivity }(\mathrm{S} / \mathrm{cm})\end{array}$ & $\begin{array}{c}\text { Ktot } \\
\left(\mathbf{W ~ m}^{-1} \mathbf{K}^{-1}\right)\end{array}$ & $\begin{array}{c}n_{\mathrm{H}} \\
\left(10^{21} \mathrm{~cm}^{-3}\right)\end{array}$ \\
\hline$x=0.01$ & $\mathrm{Ge}_{0.99} \mathrm{Ti}_{0.01} \mathrm{Te}$ & 29.79 & 6332.23 & 6.54 & 1.21 \\
\hline$x=0.03$ & $\mathrm{Ge}_{0.97} \mathrm{Ti}_{0.03} \mathrm{Te}$ & 36.45 & 4221.99 & 5.53 & 2.13 \\
\hline$x=0.05$ & $\mathrm{Ge}_{0.95} \mathrm{Ti}_{0.05} \mathrm{Te}$ & 30.23 & 3753.34 & 4.49 & 2.51 \\
\hline$x=0.07$ & $\mathrm{Ge}_{0.93} \mathrm{Ti}_{0.07} \mathrm{Te}$ & 32.70 & 3942 & 4.09 & 3.20 \\
\hline
\end{tabular}

